\title{
Efeitos da sucessão florestal sobre a anurofauna (Amphibia: Anura) da Reserva Catuaba e seu entorno, Acre, Amazônia sul-ocidental
}

\author{
Vanessa M. de Souza '; Moisés B. de Souza ${ }^{2} \&$ Elder F. Morato ${ }^{2}$ \\ ${ }^{1}$ Avenida Assis Chateaubriand 1170, ap. 304, Edifício Azul, Setor Oeste, 74130-011 Goiânia, Goiás, Brasil. \\ E-mail: phyllomedusavms@yahoo.com.br \\ ${ }^{2}$ Departamento de Ciências da Natureza, Universidade Federal do Acre. Rodovia BR-364, km 04, Distrito Industrial, \\ 69915-900 Rio Branco, Acre, Brasil. E-mail: moisebs@terra.com.br; elderfmorato@yahoo.com.br
}

\begin{abstract}
Effect of the forest succession on the anurans (Amphibia: Anura) of the Reserve Catuaba and its periphery, Acre, southwestern Amazonia. The objective of this work it was verify the abundance, richness, and the anuran composition in plots of vegetation of different succession stages in a forest and the matrix that surrounds it, of Acre $\left(10^{\circ} 04^{\prime} \mathrm{S}, 67^{\circ} 37^{\prime} \mathrm{W}\right)$. The sampling was carried out between August 2005 and April 2006 in twelve plots located in three different sites in the forest. In each site four kinds of environments were chosen: primary forest (wood), secondary forest (capoeira), periphery (matrix) and secondary forest (succession). A total of twenty-seven species distributed in seven families was found. Greater abundance was registered in the plots of matrix two and capoeira three and the least in succession one. The richness was greater in matrix two, with the greater number of exclusive species. The abundance of anurans correlated significantly, with the average circumference at the breast height of the trees of the plots. The richness however correlated only marginally, with this structural feature. The larger richness in plots of capoeira and matrix can be explained partially by the existence of temporary ponds and more structural heterogeneity is able constitute intermediary stages in a gradient of perturbation and this can increase the biodiversity. Thus environments with intermediary levels of disturbance are important for the conservation of the diversity of anuran amphibians.
\end{abstract}

KEY WORDS. Cronosequence; diversity; perturbation; structure of vegetation; richness of anuran.

RESUMO. O trabalho teve como objetivo verificar a abundância, riqueza e a composição da anurofauna em diferentes estágios sucessionais em uma floresta do Acre $\left(10^{\circ} \mathrm{O} 4^{\prime} \mathrm{S}, 67^{\circ} 37^{\prime} \mathrm{W}\right)$ e seu entorno. Os dados foram obtidos entre agosto de 2005 a abril de 2006 em doze parcelas, localizadas em três áreas diferentes da floresta. Em cada área foram escolhidos quatro tipos de ambientes: floresta primária (mata), floresta secundária (capoeira), entorno (matriz) e floresta secundária (sucessão). Observou-se a presença de 27 espécies distribuídas em sete famílias. Maior abundância foi constatada na matriz dois e capoeira três, e a menor na sucessão um. A maior riqueza foi constatada na matriz dois, com o maior número de espécies exclusivas. $\mathrm{A}$ abundância de anuros correlacionou-se significativamente com a circunferência das árvores e lianas. A riqueza de anuros correlacionouse marginalmente com a circunferência das árvores. A maior riqueza em ambientes de capoeiras e matriz pode ser explicada em grande parte pela existência nesses locais de poças d'água, maior heterogeneidade estrutural e poderem constituir estágios intermediários de perturbação. Esses estágios têm sido apontados como fatores que promovem e mantêm níveis elevados de biodiversidade. Ambientes com níveis intermediários de perturbação são importantes para a conservação da anurofauna.

PALAVRAS-CHAVE. Cronoseqüência; diversidade; estrutura da vegetação; perturbação; riqueza de anuro.

A estrutura física dos ambientes afeta a riqueza e composição da sua biota (Schoener 1970, Ishitani et al. 2003). Nos ambientes terrestres vários componentes da estrutura da vegetação têm sido empregados na predição de características da sua fauna (Pianka 1967, August 1983, FloAater \& Zalucki 2000, VidAL \& CinTra 2006). Freqüentemente, a estrutura da vegetação tem sido empregada nesse tipo de abordagem em termos de complexidade e heterogeneidade (August 1983). O tipo e intensidade de respostas dos animais dependem do grupo taxonômico em questão (Terborgh \& Petren 1991).

A complexidade e heterogeneidade da vegetação podem ser responsáveis por diferenças entre os ambientes, em relação à produtividade primária, disponibilidade de recursos e microhabitats, interações interespecíficas e diferenças microclimáticas (Connell \& Slatyer 1977, Sousa 1984, Pickett \& White 1985).

Revista Brasileira de Zoologia 25 (1): 49-57, March, 2008 
Eventos de perturbação nos ambientes terrestres podem promover o processo de sucessão secundária da vegetação, o qual altera a composição florística e estrutura da vegetação local (Sousa 1984). Isso, por sua vez, pode alterar a fauna tanto de invertebrados (Richards 1926, Schoener 1970, Brown \& Southwood 1987, Brown 1991, StefFan-Dewenter \& TscharntKe 2001) como de vertebrados (Richards 1926, Fonseca 1989, Nichols \& Nichols 2003). Os efeitos da sucessão florestal sobre a estrutura das assembléias de anuros são muito pouco conhecidos, em particular no bioma amazônico (Tocher 1998).

Atualmente existem no mundo cerca de 5.362 espécies de anfíbios anuros, distribuídas entre 45 famílias (Frost 2007). Cerca de $45 \%$ dessas espécies ocorrem na América tropical (Duellman 1978). No Brasil existem 776 (SBH 2005), espécies distribuídas em 10 famílias e somente na última década foram descritas no país cerca de 60 espécies, o que sugere que a diversidade desse grupo deve ser ainda maior (CAldwell 1991, Heyer \& Hardy 1991, Eterovick \& Sazima 1998).

No Estado do Acre existem cerca de 126 espécies, distribuídas em oito famílias (Souza et al. 2003). Essa diversidade é grande, em relação a outros biomas (CARDoso \& Souza 1996, Souza \& Cardoso 2002, Souza et al. 2003). Contudo, pouco se conhece sobre a biologia e ecologia do grupo nessa região (Bernarde et al. 1999, Souza et al. 2002, Souza \& Haddad 2003).

Os anfíbios são muito sensíveis as modificações no habitat, poluentes e a mudanças climáticas globais (PHILLIPS 1990, Alford \& Richards 1999). Estas perturbações têm provocado declínio e até mesmo extinção local de suas populações no sudeste do Brasil (Heyer et al. 1988). Por isso, são considerados excelentes bioindicadores de alterações ambientais (PHILLIPS 1990, VitT et al. 1990) e têm adquirido grande relevância nas avaliações ecológicas (HeYER et al. 1988).

O presente trabalho teve como objetivo verificar a abundância, a riqueza e a composição da anurofauna em ambientes de diferentes estágios sucessionais de cronoseqüências de uma floresta do Acre e seu entorno. Mais especificamente pretende testar as seguintes hipóteses: 1) ambientes menos perturbados e em estágios sucessionais mais avançados possuem maior riqueza e abundância de anuros; 2) a riqueza, composição florística e a estrutura da vegetação afetam a abundância, riqueza e composição de anuros; e 3) a complexidade e a heterogeneidade estruturais da vegetação são preditoras da abundância e riqueza de espécies.

Este trabalho faz parte de um estudo maior cujo objetivo principal é verificar os efeitos da sucessão florestal sobre a fauna, de invertebrados (Morato \& MarTins 2005) e representa o primeiro esforço no sentido de investigar esses efeitos sobre um grupo de vertebrados na Amazônia Sul-Ocidental.

\section{MATERIAL E MÉTODOS}

O trabalho foi realizado na Reserva Experimental Catuaba (REC) $\left(10^{\circ} 04^{\prime} \mathrm{S}, 67^{\circ} 37^{\prime} \mathrm{W}\right.$ e altitude média de $\left.214 \mathrm{~m}\right)$. A REC pertence à Universidade Federal do Acre (UFAC) e localiza-se próxima ao entroncamento das Rodovias BR-364 e 317 no município de Senador Guiomard, Acre.

A área, como um todo, consiste de um fragmento florestal de vegetação, com cerca de 2.111 ha, isolado de porções de floresta primária aproximadamente por distâncias variáveis entre 0,8 e 7,4 km. Sua vegetação é constituída principalmente por floresta tropical de terra firme e inclui floresta aberta com bambus e palmeiras (forma predominante), floresta densa, florestas secundárias (capoeiras) e pastagens. O sub-bosque é muito fechado e possui predomínio de cipós e bambus. O dossel é aberto e possui altura variando entre 20-40 m. O relevo é suavemente ondulado salvo próximo a rede de drenagem a qual é constituída por nove igarapés. Os principais tipos de solo são latossolo vermelho e podzólico vermelho (ACre 1991, Morato \& Martins 2005).

A matriz da paisagem que envolve o entorno da REC é o resultado do processo comum de uso da terra na região (FunTAC 1990, ACRE 1991, 2000): bordas de floresta, pastagens, campos agrícolas, pomares, habitações e estradas.

A temperatura média anual na região varia entre 22 a $24^{\circ} \mathrm{C}$. Contudo, nos períodos de mais frios (em geral, no mês de agosto) a temperatura pode variar entre 12 a $14^{\circ} \mathrm{C}$. O total médio de precipitação anual é de $1.973 \mathrm{~mm}$, sendo janeiro o mês mais chuvoso com uma média de 298,6 mm e julho o menos com 25,2 mm (Mesquita \& Paiva 1995, Mesquita 1996). Há três estações definidas, em relação à precipitação: 1) estação chuvosa (dezembro, janeiro, fevereiro e março $=55,8 \%$ das chuvas); 2 ) estação seca (junho, julho e agosto = 5,7\%); e 3) estação intermediária (setembro, outubro, novembro, abril e maio $=38,5 \%$ ).

Os anuros foram amostrados em 12 parcelas de aproximadamente 1 ha, localizadas em três locais diferentes da REC. Em cada local foram escolhidos quatro tipos de ambientes (tratamentos): 1) floresta primária, aqui denominada de mata, a qual representa a área menos perturbada e em estágio sucessional mais avançado e constitui a floresta típica da região; 2) floresta secundária, aqui denominada capoeira, a qual também está em processo sucessional, porém mais lento; 3) entorno, aqui denominado de matriz, a qual é constituída por áreas desmatadas, em geral fora do perímetro da reserva, ocupadas por fazendas que possuem pastagens, plantações, habitações humanas, bordas de floresta e corpos d'água de tamanhos variáveis e 4) floresta secundária, aqui denominada sucessão, a qual foi experimentalmente desmatada na estação seca de 2001, queimada, limpa, pulverizada de forma homogênea com herbicida e deixada em regeneração.

Cada área constituiu uma unidade amostral. Portanto, cada conjunto de áreas de diferentes ambientes representa um gradiente sucessional: mata, capoeira, sucessão e matriz. Cada tipo de ambiente (tratamento) foi repetido três vezes. Dessa forma, cada tratamento e suas respectivas repetições foram designados como: mata 1 , mata 2 , mata 3 , capoeira 1 , capoeira 2 , capoeira 3 , matriz 1 , matriz 2 , matriz 3 , sucessão 1 , sucessão 2 e sucessão 3 . Ambientes diferentes, mas com o mesmo núme- 
ro, pertencem à mesma repetição ou conjunto dentro da REC. Cada repetição equivale a uma cronoseqüência (TERBorgh \& Petren 1991) ou gradiente, em relação aos estágios de sucessão da vegetação.

Áreas de uma mesma repetição situam-se mais próximas entre si do que de repetições diferentes. As distâncias entre as áreas variam de aproximadamente 107 a $6.430 \mathrm{~m}$. As áreas da repetição 1 são mais distantes entre si do que as das outras repetições e as da repetição 3 são as mais próximas. O conjunto de áreas da repetição 1 é também o mais distante dos demais.

Cada uma das áreas, independentemente do tipo de estrutura da sua vegetação, foi dividida, por trilhas de aproximadamente um metro de largura, em 12 sub-parcelas de 30 × 20 m. As trilhas foram feitas com a finalidade de facilitar a locomoção dos pesquisadores, tendo em vista que, tanto nas florestas secundárias como nas primárias do Acre, o sub-bosque é muito fechado e de difícil trânsito.

As amostragens foram realizadas num período de nove meses entre agosto de 2005 e abril de 2006, totalizando 22 dias e cerca de 126 horas. Os dados foram tomados através de observações visuais e auditivas, por meio de caminhadas diurnas e noturnas através das parcelas. Nas matrizes as caminhadas foram feitas ao acaso e nas demais áreas foram sistematizadas ao longo das trilhas. O esforço amostral em cada área foi de 1h30min. Alguns espécimes foram capturados, fotografados, sacrificados, fixados em solução de formalina a 10\% e posteriormente transferidos para solução alcoólica a 70\% e depositados na Coleção de anfíbios da Universidade Federal do Acre, servindo como material testemunho.

Os dados climatológicos correspondentes ao período de amostragem foram obtidos na Estação de Meteorologia do Campus da UFAC, Rio Branco (Tab. I).

Em cada parcela, concomitantemente a amostragem de anuros, foram medidas treze variáveis a fim de caracterizar a estrutura geral da vegetação: altura das árvores, circunferência á altura do peito (CAP) de árvores e lianas (CAP $\geq 8 \mathrm{~cm})$, densidade de árvores, densidade de caules, área basal, cobertura do dossel, densidade de colmos de bambus, densidade de arbustos (altura $\geq 0,5 \mathrm{~m}$ ), altura de arbustos, densidade de troncos no chão e riqueza florística. Considerou-se como árvore todo elemento lenhoso com altura igual ou superior a $3 \mathrm{~m}$ e como arbusto toda forma vegetal entre 0,5 e $3 \mathrm{~m}$ de altura.

A partir dos valores de todas essas variáveis foi gerado um índice operacional de complexidade e outro de heterogeneidade estrutural da vegetação de cada parcela a partir de August (1983) e Waddington et al. (1986). Cada parcela foi ranqueada em relação aos valores médios para cada variável estrutural. Não foram atribuídos pesos diferentes às variáveis. As parcelas com os valores mais baixos receberam escores menores. Após, os escores de cada variável foram somados para cada parcela. Essa quantidade final foi denominada "índice de complexidade estrutural" da vegetação. Portanto, a parcela com o maior valor desse índice foi aquela com valores elevados para a maior parte das variáveis consideradas. Procedimento semelhante foi adotado para o cômputo do "índice de heterogeneidade estrutural" da vegetação. Contudo, neste caso cada parcela foi ranqueada em relação aos valores das variâncias de cada variável. De forma similar, a parcela mais heterogênea foi, portanto, a que apresentou o maior valor dessa soma de escores. Neste trabalho, uma parcela com grande complexidade estrutural é aquela com valores elevados para as variáveis consideradas da vegetação. Por outro lado, uma parcela com grande heterogeneidade é aquela com uma grande variação em relação a essas mesmas variáveis. A complexidade aqui considerada é determinada, portanto, pela magnitude de qualquer variável da vegetação e a heterogeneidade pela magnitude de sua variação.

As análises estatísticas foram realizadas através dos programas Statistica 5.0 (1995), BioDiversity Professional 2.0 (1997) e Bioestat 2.0 (2000) e foram baseadas em Ludwing \& REYNOLDS (1988), Siegel \& Castellan (1988) e Sokal \& Rohlf (1995). Para todas as análises considerou-se $\alpha=0,05$.

As analises de correlações foram realizadas através do coeficiente de Pearson. Os padrões de riqueza de espécies de anuros obtidos em cada cronoseqüência foram correlacionados através do coeficiente de concordância de Kendall.

Tabela I. Dados Climatológicos obtidos durante o período de amostragem do trabalho.

\begin{tabular}{lccccc}
\hline Mês/ano & Temperatura mínima $\left(\mathrm{C}^{\circ}\right)$ & $\begin{array}{c}\text { Temperatura máxima } \\
\left(\mathrm{C}^{\circ}\right)\end{array}$ & $\begin{array}{c}\text { Temperatura média } \\
\left(\mathrm{C}^{\circ}\right)\end{array}$ & $\begin{array}{c}\text { Precipitação total } \\
(\mathrm{mm})\end{array}$ & $\begin{array}{c}\text { Umidade relativa } \\
(\%)\end{array}$ \\
\hline Agosto/2005 & 18,4 & 34,6 & 25,1 & 0 & 71,0 \\
Setembro & 18,1 & 34,2 & 25,0 & 23,2 & 69,0 \\
Outubro & 22,2 & 33,5 & 26,5 & 195,3 & 81,0 \\
Novembro & 22,4 & 31,7 & 25,9 & 130,9 & 83,0 \\
Dezembro & 22,5 & 30,8 & 25,6 & 338,8 & 90,2 \\
Janeiro/2006 & 22,5 & 30,7 & 25,5 & 270,2 & 90,0 \\
Fevereiro & 22,7 & 30,4 & 25,5 & 371,9 & 92,0 \\
Março & 22,9 & 31,7 & 25,9 & 181,7 & 89,0 \\
Abril & 22,1 & 30,6 & 25,2 & 230,0 & 90,0 \\
\hline
\end{tabular}


A similaridade em relação à composição faunística foi analisada através da geração de uma matriz de similaridade empregando o índice binário de Jaccard. Os valores obtidos foram posteriormente empregados em uma amalgamação através do método de agrupamento por pares de médias não ponderadas (UPGMA).

\section{RESULTADOS}

Foram registradas 27 espécies, distribuídas em sete famílias: Aromobatidae (3), Brachycephalidae (2) Bufonidae (2), Dendrobatidae (2), Hylidae (10), Leptodactylidae (7) e Microhylidae (1) (Tab. II). As espécies mais abundantes foram Leptodactylus andreae (Muller, 1923) (27\%), Eleutherodactylus conspicillatus (Günther, 1858) (19\%) e Rhinella margaritifer (Laurenti, 1768) (10\%). Leptodactylus andreae ocorreu em todas as parcelas e Eleutherodactylus conspicillatus somente não foi registrada na matriz 2. A nomenclatura utilizada segue Frost (2007).

Maior abundância foi constatada na matriz 2 e capoeira 3 , e a menor na sucessão 1 . A maior riqueza foi constatada na matriz 2 (12 espécies), matriz 3 (9), capoeira 2 (9) e capoeira 3 (8). Na matriz 2 também foi encontrado o maior número de espécies exclusivas (6). A abundância de registros nas parcelas correlacionou-se significativamente com a sua riqueza em espécies $(\mathrm{r}=0,862 ; \mathrm{p}=0,0003 ; \mathrm{g} .1 .=10)$. De modo geral, as espécies mais abundantes foram as que também ocorreram em maior número de parcelas $(r=0,9546 ; p=0,000 ;$ g.l. $=25)$. Cerca de $48 \%$ do total de espécies foram registradas uma única vez.

Nas três cronoseqüências o menor número de espécies foi constado nas parcelas de sucessão e o maior nas parcelas de matriz e capoeira (Fig. 1). O número de espécies registrados nas parcelas de mata também foi pequeno nas três cronoseqüências. Houve
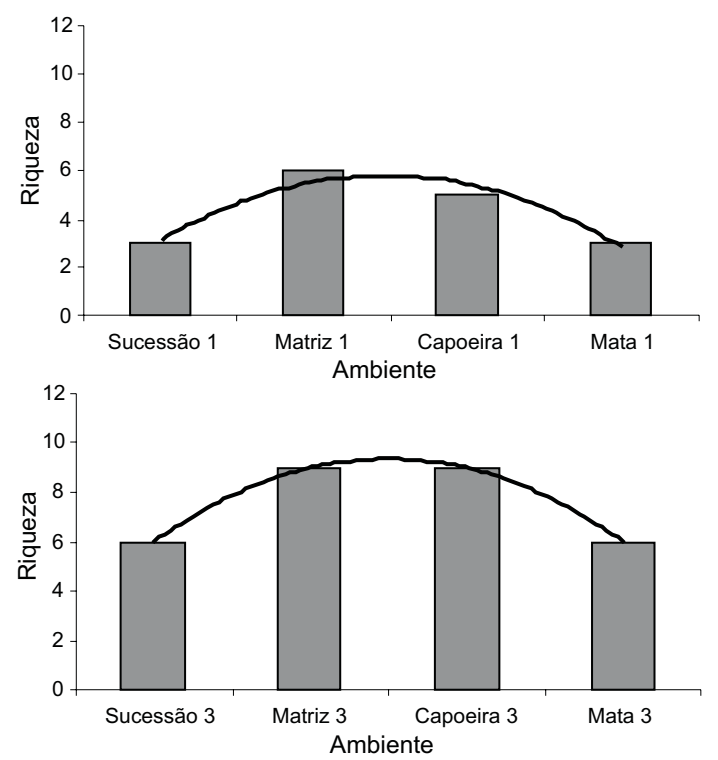

Figura 1. Cronoseqüências e parcelas amostradas durante o trabalho. uma concordância significativa $(\mathrm{W}=3,649 ; \mathrm{p}=0,0000 ;$ g.l. $=3$ ) entre as três cronoseqüências, em relação aos padrões de riqueza das parcelas. Resultado semelhante foi obtido em relação à abundância de registros ( $\mathrm{W}=5,689 ; \mathrm{p}=0,0000 ;$ g.l. = 3).

Um dendrograma de similaridade (índice de Jaccard) em relação á composição da anurofauna entre as parcelas é mostrado na figura 2 . As espécies registradas na mata 1 foram as mesmas que as registradas na sucessão 1 . A matriz 2 apresentou o menor valor de similaridade em relação as outras parcelas. De modo geral, as parcelas da cronoseqüência 1 apresentaram pequena similaridade com parcelas das outras cronoseqüências. As parcelas de mata apresentaram entre si maior similaridade média $(39,7 \%)$ do que as parcelas de capoeira $(37,3 \%)$, sucessão $(37,1 \%)$ e matriz $(28,8 \%)$.

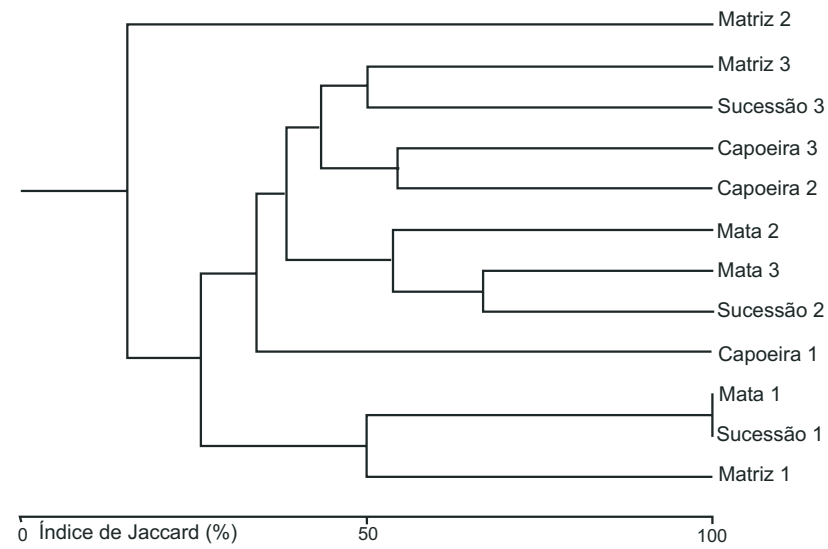

Figura 2. Dendrograma de similaridade (índice de Jaccard) em relação à composição da anurofauna das parcelas amostradas.

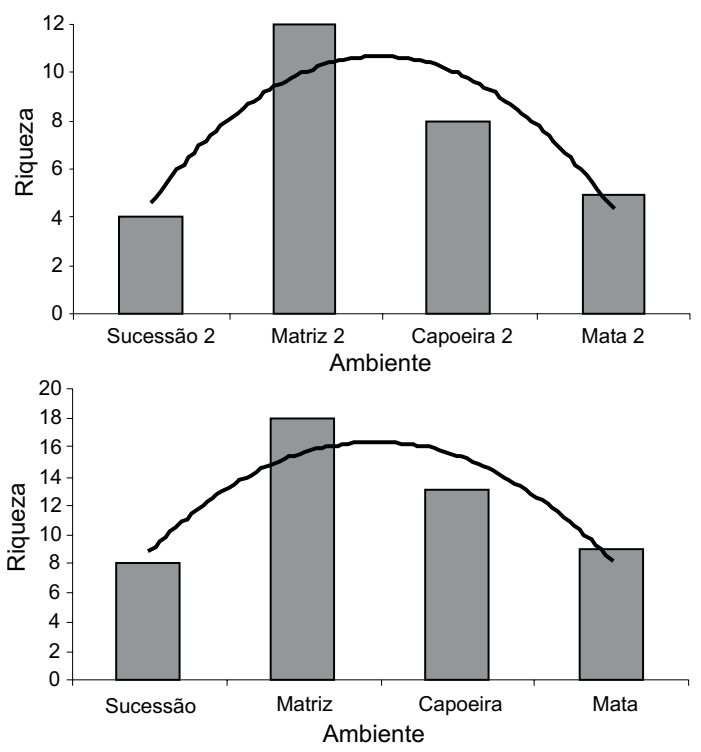


Tabela II. Lista das espécies de anfíbios anuros encontrados na Reserva Catuaba (AC) durante o período de agosto de 2005 a abril de 2006 e parcelas utilizadas durante o estudo. (MTZ1) Matriz 1, (SUC1) sucessão 1, (CAP1) capoeira 1, (MAT1) mata 1, (MTZ2) matriz 2, (SUC2) sucessão 2, (CAP2) capoeira 2, (MAT2) mata 2, (MTZ3) matriz 3, (SUC3) sucessão 3, (CAP3) capoeira 3, (MAT3) mata 3.

Espécies MTZ1 SUC1 CAP1 MAT1 MTZ2 SUC2 CAP2 MAT2 MTZ3 SUC3 CAP3 MAT3 Total

Aromobatidae

Allobates femoralis (Boulenger, 1884)

Allobates sp.

Allobates marchesianus (Melin, 1941) MTZ1 SUC1 CAP1 MAT1 MTZ2 SUC2 CAP2 MAT2 MTZ3 SUC3 CAP3 MAT3 Total

Brachycephalidae

Eleutherodactylus conspicillatus (Günther, 1858)

Eleutherodactylus gr. lacrimosus (Jiménez de la Espada, 1875)

$\begin{array}{llll}1 & 2 & 2 & 3\end{array}$

Eleutherodactylus sp.

Bufonidae

Chaunus marinus (Linnaeus, 1758)

Rhinella gr. margaritifer (Laurenti, 1768)

Dendrobatidae

Ameerega hahneli (Boulenger, 1884)

Ameerega trivittata (Spix, 1824)

Hylidae

Dendropsophus acreanus (Bokermann, 1964)

Dendropsophus gr. minutus Peters, 1872

Dendropsophus leucophyllatus (Beireis, 1783)

Hypsiboas granosus (Boulenger, 1882)

Hypsiboas lanciformis (Cope, 1871)

Hypsiboas punctatus (Schneider, 1799)

Phyllomedusa camba De La Riva, 1999

Scinax garbei (Miranda-Ribeiro, 1926)

Scinax ruber (Laurenti, 1768)

Trachycephalus venulosus (Laurenti, 1768)

Leptodactylidae

Leptodactylus andreae (Muller, 1923)

Leptodactylus sp.

Leptodactylus bolivianus Boulenger, 1898

Leptodactylus pentadactylus (Laurenti, 1768)

Leptodactylus petersii (Steindachner, 1864)

Leptodactylus rhodomystax Boulenger, 1884 "1883"

\begin{tabular}{|c|c|c|c|c|c|c|c|c|c|c|c|c|}
\hline & & 1 & & & & & & & & & & 1 \\
\hline & & & & & & & & & & & 1 & 1 \\
\hline \multirow[t]{12}{*}{4} & & & & 3 & & & & 1 & & & & 8 \\
\hline & & & & 2 & 3 & 1 & 2 & 2 & 1 & 1 & 2 & 14 \\
\hline & & & & 1 & & & & & & & & 1 \\
\hline & & & & & & & & & & 1 & & 1 \\
\hline & & & & 1 & & & & & & & & 1 \\
\hline & & & & 2 & & & & 1 & & & & 3 \\
\hline & & & & 2 & & & & & & & & 2 \\
\hline & & & & 1 & & & & & & & & 1 \\
\hline & & & & & & & & & 1 & & & 1 \\
\hline & & & & 1 & & & & & & & & 1 \\
\hline & & & & 1 & & & & & & & & 1 \\
\hline & & & & 2 & & & 2 & & & & & 4 \\
\hline 1 & 1 & & 1 & 1 & & 1 & & & & & & 5 \\
\hline 1 & & & & & & & & & & & & 1 \\
\hline \multirow[t]{6}{*}{4} & 2 & 2 & 5 & 3 & 3 & 1 & 4 & 2 & 3 & 5 & 2 & 36 \\
\hline & & 3 & & & & 2 & 1 & 1 & & 1 & 1 & 9 \\
\hline & & 1 & & & & & & & & & & 1 \\
\hline & & & & & & 1 & & 1 & 1 & 2 & & 5 \\
\hline & & & & & & & & 1 & 1 & & & 2 \\
\hline & & & & & & 1 & & & & 1 & & 2 \\
\hline 1 & & & & & & & & & & & & 1 \\
\hline 12 & 5 & 9 & 9 & 20 & 10 & 12 & 12 & 11 & 9 & 16 & 9 & 134 \\
\hline 6 & 3 & 5 & 3 & 12 & 4 & 8 & 5 & 9 & 6 & 9 & 6 & 27 \\
\hline 2 & & 2 & & 6 & & & & & 1 & 2 & 1 & 14 \\
\hline
\end{tabular}

Microhylidae

Elachistocleis ovalis (Schneider, 1799)

Total de registros

Riqueza de espécies

Riqueza de espécies exclusivas 
A abundância de anuros correlacionou-se significativamente com a circunferência das árvores e lianas (CAP), ( $r=0,670$; $\mathrm{p}=0,017$; g.l. = 10). No entanto, a abundância não correlacionouse com a complexidade, nem com a heterogeneidade da vegetação.

A riqueza de anuros correlacionou-se marginalmente com a circunferência das árvores (CAP), das parcelas amostrais ( $\mathrm{r}=$ 0,$569 ; \mathrm{p}=0,053$; g.l. = 10) de uma maneira semelhante à abundância. E não houve correlação entre riqueza, complexidade e heterogeneidade, no ambiente.

\section{DISCUSSÃO}

A única variável da estrutura da vegetação que individualmente correlacionou-se com a abundância e riqueza de anuros foi á circunferência das árvores e lianas (CAP). Os índices de complexidade e heterogeneidade não apresentaram correlação significativa com a abundância e riqueza de anuros. Entretanto, a abundância e a riqueza de anuros das áreas podem ser explicadas, pelo menos em parte, pela estrutura geral da vegetação, uma vez que os sapos foram mais comuns na área de matriz 2. Isso sugere que esses parâmetros das assembléias de anuros devem sofrer a influência conjunta de um grande número de variáveis que não apenas as consideradas neste trabalho, em especial da vegetação. Por outro lado, nenhuma dessas variáveis possui uma relação direta com a existência de corpos d'água, os quais foram mais comuns na matriz 2 e são provavelmente o componente do ambiente mais importante para a reprodução desses animais.

GiaretTa et al. (1999) mostrou que a abundância de anuros da serapilheira em uma floresta do sudeste do Brasil corrrelacionou-se positivamente com a cobertura do solo por serapilheira, com a profundidade da camada de serapilheira no solo, massa seca de serapilheira e a densidade de troncos no solo. A altitude também correlacionou-se positivamente com a densidade e a biomassa dos anuros.

Allmon (1991) em seu estudo na Floresta Amazônica mostrou que a densidade de anuros da serapilheira varia muito entre florestas tropicais do mundo todo. Este fato pode estar relacionado às variações na disponibilidade de nutrientes e características de diferentes tipos de solo, juntamente com as idades das florestas em questão e, portanto, com o estágio sucessional. A abundância e a riqueza de anuros foram comparadas com variáveis da estrutura da vegetação, inclusive com a espessura da serapilheira. Contudo, essa variável não correlacionou-se com a abundância de anuros. A abundância e a diversidade dentro das parcelas foram fortemente sazonais e apresentaram um pico no final da estação úmida. As abundâncias de árvores, ervas e palmeiras não correlacionou-se com a abundância geral de anuros. A abundância de troncos no chão e a topografia também não explicaram a abundância de anuros. A massa de serapilheira também não explicou a abundância de anuros. Portanto, isso mostra que variáveis do ambiente tomadas isoladas talvez não sejam tão importantes como preditoras da ocorrência de anuros pelo menos as associadas á estrutura da vegetação.
Poucos estudos têm abordado os efeitos da sucessão sobre as espécies de animais e outros organismos. A maioria enfatiza as assembléias de vertebrados como mamíferos, aves e lagartos (Margalef 1977, Brown \& Southwood 1987, FonseCa 1989, Brown 1991, Nichols \& Nichols 2003). Contudo, algumas características específicas do ambiente podem influenciar as assembléias de anuros, tais como a disponibilidade de determinados microhábitats e poças d'água.

Tocher et al. (1997) investigou na Amazônia Central os efeitos da fragmentação florestal sobre a comunidade de anuros por um período de 10 anos. Após o isolamento, os maiores fragmentos apresentavam maior número de espécies e a riqueza diminuiu com o tamanho do fragmento. Contudo, algumas espécies foram mais comuns em fragmentos de um tamanho do que de outro. Outras ainda apresentaram uma ocorrência muito menor em áreas de floresta primária contínua do que em fragmentos de menores tamanhos. Individualmente, portanto, as respostas podem ser muito idiossincráticas e dependentes da espécie.

A riqueza de espécies encontrada no presente estudo (27) foi relativamente elevada, quando comparada com a obtida (31) no único levantamento de anfíbios realizado anteriormente para toda a Reserva Catuaba (CARDoso \& Souza 1996). O número encontrado no presente trabalho foi ligeiramente menor possivelmente por ter sido realizado em apenas algumas parcelas localizadas dentro da reserva e em seu entorno, diferentemente do realizado por CARDoso \& Souza (1996), no qual, as amostragens foram realizadas em toda a reserva. No entanto, Cardoso \& SouZA (1996) não informaram o esforço amostral realizado em seu estudo o que impossibilita uma comparação mais segura.

Todas as espécies aqui registradas são conhecidas para o Estado do Acre (Souza \& Cardoso 2002). No entanto, três entidades não tiveram suas identificações ao nível de espécie precisamente definidas (Allobates sp., Eleutherodactylus sp. e Leptodactylus sp.). O maior número de espécies foi constatado na matriz 2, possivelmente por apresentar uma poça temporária, a qual é sítio de reprodução para várias espécies.

A maior riqueza em ambientes de capoeira e matriz pode ser explicada em grande parte pela existência nesses locais de poças d'água, pela sua maior heterogeneidade estrutural e pelo fato desses ambientes poderem constituir estágios intermediários de perturbação (ConNell 1978, SousA 1984, Huston 1994, RosenZWEIg 1995, ToKeshi 1999). Mudanças na composição das assembléias de vários grupos de animais, vertebrados e invertebrados, ao longo da sucessão da vegetação também vêm sendo relatadas em diferentes biomas (Richards 1926, MARgalef 1977, Sousa 1984, Usher \& JefFerson 1991, Thomas 1995, SteffanDewenter \& TscharntKe 1997, Balmer \& Erhardt 2000, Imbeau et al. 2003, Nichols \& Nichols 2003).

Leptodactylus andreae foi encontrada em todos os ambientes, sendo considerada generalista, corroborando outros estudos na Amazônia (Tocher et al. 1997, Tocher 1998, BRANDÃo 2002). 
Em ambientes menos perturbados provavelmente algumas espécies excluem outras, reduzindo a diversidade (TownsEND et al. 2006). Os ambientes mais perturbados serão colonizados por espécies pioneiras que são mais adaptadas a essa situação (White \& Pickett 1985, Rosenzweig 1995, Lertzman \& Fall 1998). Ambientes heterogêneos devem promover maior diversidade, uma vez que favorecem a coexistência de espécies com diferentes graus de especialização. Uma explicação adicional para a alta diversidade nesses ambientes é a hipótese dos níveis intermediários de perturbação, segundo a qual a freqüência e intensidade de distúrbios afetam os padrões de diversidade. Um nível moderado de perturbação favoreceria tanto espécies de estágios sucessionais avançados como pioneiras, o que resultaria em uma alta diversidade geral de espécies (Grime 1973, HorN 1975, Sousa 1984, White \& Pickett 1985, Rosenzweig 1995, LerTZMAN \& Fall 1998, TOKESHi 1999).

Níveis intermediários de perturbação têm sido apontados como fatores que promovem e mantêm níveis elevados de biodiversidade (FonsECA 1989, BRown 1991). Ambientes que são mais heterogêneos espacialmente podem acomodar mais espécies, porque eles provêm uma maior quantidade de microhábitats, uma gama maior de microclimas e mais esconderijos as presas, aumentando assim a diversidade de recursos (Townsend et al. 2006). Além disso, a heterogeneidade espacial promoveria uma maior variação espacial também nos fatores abióticos do ambiente o que poderia favorecer no final uma maior riqueza de espécies (Townsend et al. 2006).

GASCON et al. (1999) também encontraram resultados semelhantes com grupos de vertebrados na Amazônia Central. Seu estudo chamou a atenção para a importância de áreas de matriz e pequenos fragmentos de floresta para a manutenção da diversidade de vertebrados e contemplou quatro grupos de animais: aves, mamíferos de pequeno porte, anfíbios e formigas. As espécies mais abundantes na matriz foram os mamíferos de pequeno porte e os sapos, que aumentaram depois do isolamento do fragmento, enquanto que aves e formigas diminuíram. Para todos os quatro grupos uma alta proporção de espécies de floresta primária foi encontrada em ambientes de matriz. Os três grupos de vertebrados exibiram correlações positivas e significativas entre abundância na matriz e vulnerabilidade á fragmentação florestal. Isso sugere que espécies que evitam a matriz tendem a se extinguir ou apresentarem uma pequena abundância nos fragmentos, enquanto aquelas que toleram ou exploram a matriz permanecem frequentemente estáveis ou apresentam uma abundância relativamente maior do que as outras.

Resultados encontrados por Morato \& Martins (2005), na mesma região, em um estudo sobre vespas e abelhas solitárias são parecidos com os aqui encontrados, mostrando que áreas de capoeira e matrizes por apresentarem uma maior heterogeneidade da vegetação também possuem maior riqueza desses organismos. Isso explica, em parte, uma maior diversidade de espécies.
No presente estudo, as matrizes não constituem gradientes naturais de sucessão, mas as mesmas podem estar funcionando como ambientes estruturalmente intermediários, por possuírem árvores frutíferas, bordas de floresta, árvores remanescentes o que acarreta uma maior heterogeneidade do ambiente e favorece uma maior diversidade.

A análise de similaridade realizada mostrou que áreas amostrais de estágios sucessionais e estrutura da vegetação semelhantes não apresentaram composição da anurofauna semelhante. Isso mais uma vez mostra que a vegetação teve uma influência direta pequena sobre a composição de anuros.

A riqueza de vespas e abelhas obtidas por Morato \& Martins (2005) nas mesmas áreas amostrais do presente estudo correlacionou-se com a riqueza da anurofauna. Isso indica que possivelmente, os anuros podem funcionar como biondicadores da diversidade de outros grupos da biota, inclusive de invertebrados, ou então grupos diferentes da fauna podem responder de forma semelhante aos efeitos da perturbação e sucessão.

De modo geral, as parcelas de floresta primária foram mais semelhantes entre si em relação á composição de anuros do que as demais áreas. A estrutura da vegetação e composição florística de áreas sucessionalmente mais jovens é em grande parte influenciada pela estocasticidade, características biológicas das espécies, interações entre elas e entre os demais componentes bióticos e abióticos (Guariguata \& Ostertag 2001). Portanto, a ocupação dessas áreas pela fauna também deve ser um evento influenciado pela estocasticidade. Os únicos outros grupos faunísticos amostrados nas áreas onde foi realizado o presente trabalho foram vespas e abelhas solitárias (Morato \& MarTins 2005). Os resultados de maior riqueza presente em capoeira e matriz foram muito semelhantes aos obtidos neste trabalho para os anuros. Isso mostra que florestas secundárias e ambientes de entorno são muitos importantes para a conservação da diversidade faunísticas tanto de vertebrados como de invertebrados.

Portanto, ambientes com níveis intermediários de perturbação são importantes para a conservação da anurofauna.

\section{AGRADECIMENTOS}

Ao CNPq pela concessão da bolsa de Iniciação Científica a V.M.S e aos amigos e companheiros de campo: Alexandre S. da Cruz e Marilene V. da Silva.

\section{LITERATURA CITADA}

ACre. 1991. Atlas Geográfico Ambiental do Acre. Rio Branco, Secretaria do Meio Ambiente, Instituto de Meio Ambiente do Acre, $48 \mathrm{p}$.

ACre. 2000. Zoneamento ecológico-econômico do Acre: recursos naturais e meio ambiente. Rio Branco, Acre, SECTMA, 116p.

Alford, R.A \& S.J. Richards.1999. Global amphibian declines: A problem in applied ecology. Annual Review of Ecology and Sistematics 30: 133-165.

Revista Brasileira de Zoologia 25 (1): 49-57, March, 2008 
Allmon, W.D. 1991. A plot study of forest floor litter frogs, Central Amazon, Brazil. Journal of Ecology 7: 503-522.

August, P.V. 1983. The role of habitat complexity and heterogeneity in structuring tropical mammal communities. Ecology 64 (6): 1495-1507.

Balmer, O. \& A. Erhardt. 2000. Consequences of succession on extensively grazed grasslands for Central European butterfly communities: rethinking conservation practices. Conservation Biology 14 (3): 746-757.

Bernarde, P.S.; M.B. Souza \& M.C.N. Kokubum. 1999. Predation on Hyla minuta Peters, 1872 (Anura, Hylidae) by Ancylometes spp. (Araneae, Pisauridae). Biociências 7 (1): 199-203.

BRANDÃo, R.A. 2002. Avaliação ecológica rápida da herpetofauna nas reserves extrativistas de pedras negras e curralinho, Costa Marques, RO. Brasil Florestal 74: 61-73.

BRown, V.K. 1991. The effects of changes in habitat structure during succession in terrestrial communities, p. 141-168. In: S.S. Bell; E.D. Mccoy \& H.R. Mushinsky (Eds). Habitat Structure: the physical arrangement of objects in space. London, Chapman, 438p.

Brown, V.K. \& T.R.E. Southwood. 1987. Secondary succession: patterns and strategies, p. 315-337. In: A.J. Gray.; M.J. Crawley \& P.J. Edwards (Eds). Colonization, Succession and Stability. Oxford, Blackwell Scientific, 482p.

Caldwell, J.P. 1991. A new species of toad in the genus Bufo from Pará, Brazil, with an unusual breeding site. Papéis Avulsos de Zoologia 37 (26): 389-400.

Cardoso, A.J \& M.B. SouzA. 1996. Distribuição temporal e espacial de anfíbios anuros no Seringal Catuaba, Estado do AcreBrasil, p. 271-292. In: J.E. Pérfaus (Ed.). Herpetological Neotropical. Venezuela, Universidade dos Andes, Actas $2^{\circ}$ Congresso Latino Americano de Herpetologia, 451p.

Connell, J.H. 1978. Diversity in tropical rain forests and coral reefs. Science 199: 1302-1310.

Connell, J.H \& R.O. Slatyer. 1977. Mechanisms of succession in natural communities and their role in community stability and organization. American Naturalist 111: 1119-1144.

ConTe, C.E \& D. DE C. Rossa-Feres. 2006. Diversidade e ocorrência temporal da anurofauna (Amphibia, Anura) em São José dos Pinhais, Paraná, Brasil. Revista Brasileira de Zoologia 23 (1): 162-175.

Duellman, W.E. 1978. The biology of an equatorial herpetofauna in Amazonian Ecuador. Miscellaneous Publication of the Museum of Natural History University of Kansas 65: 1-352.

Eterovick, P.C \& I. Sazima. 1998. New species of Proceratophrys (Anura: Leptodactylidae) from southeastern Brasil. Copeia: 159-164.

FloaAter, G.J \& M.P.Zalucki. 2000. Habitat structure and egg distributions in the processionary caterpillar Ochrogaster lunifer: lessons for conservation and pest management. Journal of Applied Ecology 37: 87-99.

FonSECA, G.A.B. 1989. Small mammal species diversity in brazilian tropical primary and secondary forests of different sizes. Re- vista Brasileira de Zoologia 6 (3): 381-422.

Frost, D.R. 2007. Amphibian species of the world: an online reference. Version 5.0. Electronic Database available at: http://research.amnh.org/herpetology/amphibia/index.php [Accessed in 20/V/2007].

FUNTAC. 1990. Monitoramento da cobertura florestal do Estado do Acre: desmatamento e uso atual da terra. Rio Branco, Secretária de Indústria e Comércio, Fundação de tecnologia do Estado do Acre, 214p.

Gascon, C.; T.E. Lovejoy; R.O. Bierregaard Jr; J.R. Malcolm; P.C. Stouffer; H.L. Vasconcelos; W.F. Laurance; B. Zimmerman; M. TOCHER \& S. BORges. 1999. Matrix habitat and species richness in tropical forest remnants. Biological Conservation 91: 223-229.

Giaretta, A.A.; K.G. Facure; J.H. de M. Meyer \& N. Chemin. 1999. Diversity and abundance of litter frogs in a forest of southeastern Brazil: seasonal and altitudinal changes. Biotropica 31 (4): 669-674.

Grime, J.P. 1973. Competitive exclusion in herbaceous vegetation. Nature 242: 344-347.

GuARIguAta, M.R \& R.Ostertag. 2001. NeoTropical secondary forest succession: changes in structural and functional characteristics. Forest Ecology and Management 148: 184-206.

Heyer, W.R.; A.S. Rand; C.A.G.Cruz \& O.L.Peixoto. 1988. Decimations, extinctions, and colonizations of frog populations in southeast Brasil and their evolutionary implications. Biotropica 20: 230-235.

Heyer, W.R \& L.M. Hardy. 1991. A new species of frog of the Eleutherodactylus lacrimosus assembly from Amazonia, South America (Amphibia: Anura: Leptodactylidae). Proceedings Biological Society of Washington, 104 (3): 436-447.

Horn, H.S. 1975. Markovian properties of forest sucession, p. 196-211. In: M.L. Cody \& J.M. Diamond (Eds). Ecology and evolution of communities. Cambridge, Harvard University Press, 545p.

Huston, M.A. 1994. Biological Diversity: The Coexistence of Species on Changing Landscapes. Cambridge, Cambridge University Press, 681p.

Imbeau, L.; P. Drapeau \& M. Monkkonen. 2003. Are forest birds categorized as "edge species" strictly associated with edges? Ecography 26 (4): 514-520.

Ishitani, M.; D.J.Kotze \& J. Niemelä. 2003. Changes in carabid beetle assemblages across an urban-rural gradient in Japan. Ecography 26: 481-489.

LERTZMAN, K \& J. FALL. 1998. From forest stands to landscapes: spatial scales and the roles of disturbances, p. 339-367. In: D.L. PETERSON $\&$ V.T. PARKer (Eds). Ecological scale: theory and applications. New York, Columbia University Press, 615p.

LudWIG, J.A \& J.F.ReYNolds. 1988. Statistical ecology: a primer on methods and computing. New York, John Wiley \& Sons, $337 p$.

Margalef, R. 1977. Ecologia. Barcelona, Ediciones Omega, $2^{\text {a }}$ ed., $951 \mathrm{p}$. 
Mesquita, C.C. DE. 1996. O clima do estado do Acre. Rio Branco, Secretaria de Estado de Ciência, Tecnologia e Meio Ambiente (SECTMA), 53p.

Mesquita, C.C. DE \& R.A. Paiva. 1995. Estudos básicos das precipitações do Acre. Rio Branco, Secretaria de Estado de Planejamento, $148 \mathrm{p}$.

Morato, E.F. \& R.P. Martins. 2005. Diversidade e composição da fauna de vespas e abelhas solitárias do estado do Acre, Amazônia Sul-Ocidental, p. 11-40. In: P.M. Drumond (Ed.). Fauna do Acre. Rio Branco. Rio Branco, Edufac, Série Estudos e Pesquisas 2, 203p.

Nichols, O.G. \& F.M. Nichols. 2003. Long-term trends in faunal recolonization after bauxite mining in the Jarrah Forest of Southwestern Australia. Restoration Ecology 11 (3): 261-272.

Phillips, K. 1990. Where have all the frogs and toads gone? BioScience 40: 422-424.

PIANKA, E.R. 1967. On lizard species diversity: North American flatland deserts. Ecology 48 (3): 333-351.

Pickett, S.T.A \& P.S. White. 1985. Patch dynamics: a synthesis, p. 371-384. In: S.T.A. Pickett \& P.S. White (Eds). The ecology of natural disturbance and patch dynamics. San Diego, Academic Press, 472p.

Richards, O.W. 1926. Studies on the ecology of English heaths. III. Animal successions at Oxshott Heath, Surrey. Journal of Ecology 14: 241-281.

Rosenzweig, M.L. 1995. Species diversity in space and time. Cambridge, Cambridge University Press, 436p.

SBH. 2005. Lista de espécies de anfíbios do Brasil. Sociedade Brasileira de Herpetologia (SBH). Disponível na World Wide Web em: http://www.sbherpetologia.org.br/checklist/ anfibios.htm [Acessado em 14/IX/2005].

Schoener, T.W. 1970. Nonsynchronous spatial overlap of lizards in patchy habitats. Ecology 51 (3): 408-418.

Siegel, S \& N.J. Castellan JR. 1988. Nonparametric statistics for the behavioral sciences. New York, McGraw-Hill, $2^{\text {nd }}$ ed., 399p.

Sokal, R.R \& F.J. RoHLF. 1995. Biometry. New York, W.H. Freeman, $3^{\text {rd }}$ ed., $887 p$.

SousA, W.P. 1984. The role of disturbance in natural communites. Annual Review of Ecology and Systematics 15: 353-391.

Souza, M.B \& A.J. Cardoso. 2002. Anfíbios registrados na Reserva Extrativista do Alto Juruá, p. 101-103. In: M. C. DA CunHA \& M. B. DE Almeida (Eds). Enciclopédia da Floresta. São Paulo, Companhia das Letras, 735p.

SouzA, M.B \& C.F.B. HADDAD. 2003. Redescrition and reevaluation of the generic status of Leptodactylus dandasi (Amphibia, Anura, Leptodactylidae) and description of its unusual advertisement call. Journal of Herpetology 37 (3): 490-497.

Souza, M.B.; M. Silveira; M.R.M. Lopes; L.J.S. Vieira; E.G. Silva; A.M. Calouro \& E.F. Morato. 2003. A Biodiversidade no Estado do Acre: conhecimento atual, conservação e Perspectivas. Tecnologia e Ciência da Amazônia 3: 45-56.

Souza, M.B \& A.J. Cardoso. 2002. Anfíbios, p. 601-614. In: M.C.
Cunha \& M.B. Almeida (Eds). Enciclopédia da Floresta. São Paulo, Companhia das Letras, 735p.

Steffan-Dewenter, I \& T. TscharntKe. 1997. Early succession of butterfly and plant communities on set-aside fields. Oecologia 109: 294-302.

Steffan-Dewenter, I. \& T. Tscharntke. 2001. Sucession of bee communities on fallows. Ecography 24: 83-93.

Terborgh, J \& K. Petren. 1991. Development of hábitat structure through succession in Amazonian floodplain forest, p. 2846. In: S.S. BeLl; E.D. Mccoy \& H.R. Mushinsky (Eds). Habitat structure: the physical arrangement of objects in space. London, Chapman, 438p.

Thomas, C.D. 1995. Ecology and conservation of butterfly metapopulations in the fragmented British landscape, p. 4663. In: A.S. Pullin (Ed.). Ecology and conservation of butterflies. London, Chapman \& Hall, 363p.

Tocher, M.D. 1998. Diferenças na composição de espécies de sapos entre três tipos de floresta e campo de pastagem na Amazônia central, p. 219-233. In: C. GASCon \& P. MoutinHo (Eds). Floresta Amazônica: dinâmica, regeneração e manejo. Manaus, INPA, MCT, 373p.

Tocher, M.D.; C. Gascon \& B.L. Zimmerman. 1997. Fragmentation effects on a central Amazonian frog community: a ten-year study, p.124-137. In: W.F. LAURENCE \& R.O. BieRREgAard JR (Eds). Tropical forest remanants: ecology, management and conservation of fragmented communities. Chicago, The University of Chicago Press, 632p.

Tokeshi, M. 1999. Species coexistence: ecological and evolutionary perspectives. Oxford, Blackwell Science, 454p.

Townsend, C.R.; M. Begon \& J.L. Harper. 2006. Fundamentos em ecologia. Porto Alegre, $2^{a}$ ed., 592p.

Usher, M.B \& R.G. JefFerson. 1991. Creating new and sucessional habitats for arthropods, p. 263-291. In: N.M. ColLins \& J.A. Thомаs (Eds). The conservation of insects and their habitats. London, Academic Press, 450p.

Vidal, M.D \& R. Cintra. 2006. Effects of Forest structure components on the occurence, group size and density of groups of bare-face tamarin (Saguinus bicolor - Primates: Callitrichinae) in Central Amazonia. Acta Amazonica 36 (2): 237-248.

Vitt, L.J.; J.P. Caldwell; H.M. Wilbur \& D.C. Smith. 1990. Amphibians as harbingers of decaly. BioScience 40: 4-18.

WAdDIngton, K.D.; L.H. Herbst \& D.W. RoubiK. 1986. Relationship between recruitment systems of stingless bees and withinnest worker size variation. Journal of the Kansas Entomological Society 59 (1): 95-102.

White, P.S \& S.T.A. PicketT. 1985. Natural disturbance and patch dynamics: an introduction, p. 3-33. In: S.T.A. PICKETT \& P.S. WHITE (Eds). The ecology of natural disturbance and patch dynamics. San Diego, Academic Press, 472p.

Received in 19.VII.2007; accepted in 28.II.2008. 